\title{
Dibucaine Inhibition of Serum Cholinesterase
}

\author{
Babiker Elamin* \\ Department of Biochemistry, College of Science, King Saud University, P.O. Box 2455, Riyadh 11451, Saudi Arabia
}

Received 25 July 2002, Accepted 26 August 2002

\begin{abstract}
The dibucaine number (DN) was determined for serum cholinesterase (EC 3.1.1.8, SChE) in plasma samples. The ones with a DN of 79-82 were used, because they had the "usual" SChE variant. The enzyme was assayed colorimetrically by the reaction of 5,5'-dithiobis-[2nitrobenzoic acid] (DTNB) with the free sulfhydryl groups of thiocholine that were produced by the enzyme reaction with butrylthiocholine (BuTch) or acetylthiocholine (AcTch) substrates, and measured at $412 \mathrm{~nm}$. Dibucaine, a quaternary ammonium compound, inhibited SChE to a minimum within $2 \mathrm{~min}$ in a reversible manner. The inhibition was very potent. It had an $\mathrm{IC}_{50}$ of $5.3 \mu \mathrm{M}$ with BuTch or $3.8 \mu \mathrm{M}$ with AcTch. The inhibition was competitive with respect to BuTch with a $K_{i}$ of $1.3 \mu \mathrm{M}$ and a linear-mixed type (competitive/noncompetitive) with respect to AcTch with inhibition constants, $K_{i}$ and $K_{I}$ of 0.66 and $2.5 \mu \mathrm{M}$, respectively. Dibucaine possesses a butoxy side chain that is similar to the butryl group of BuTch and longer by an ethylene group from AcTch. This may account for the difference in inhibition behavior. It may also suggest the existence of an additional binding site, other than the anionic binding site, and of a hydrophobic nature.
\end{abstract}

Keywords: Dibucaine, Dibucaine binding site, Dibucaine number, Inhibition kinetics, Pseudocholinesterase

\section{Introduction}

Serum cholinesterase (EC 3.1.1.8, SChE), also known as pseudocholinesterase or butrylcholinesterase, is an esterase of an unknown physiological role (Masson et al., 1998). It was previously suggested to hydrolyze the choline esters that may arise in vivo, and which would inhibit acetylcholinesterase (EC 3.1.1.7, AChE) (Lehman and Silk, 1953). However, SChE was found useful in terminating the neuromuscular

*To whom correspondence should be addressed.

Tel: 96614675936; Fax: 96614675791

E-mail:belamin@ksu.edu.sa block of the muscle relaxants suxamethonium and mivacurium. It hydrolyses these compounds which are sometimes used in clinical anesthesia to facilitate intubation or other medical procedures (Goedde et at., 1968; Hersh et al., 1974; Cook et al., 1989; Ostergard et al., 1992; Fleming et al., 1996). However, some patients were suxamethonium-sensitive and quantitatively low in SChE activity, a geneticallydetermined property (Kalow and Genest, 1957; Thompson and Wittaker, 1966; Hobbiger and Peck, 1969; VibyMogensen, 1981; Cattozzo et al., 1993; Mitchell and HarropGriffiths, 1994; Sockalingam and Green, 1995). Thus, a pretest must be used to determine the SChE phenotype prior to suxamethonium or mivacurium infusion, otherwise, there is the risk of prolonged muscular relaxation and apnea. Kalow and Davis (1958), using dibucaine as an inhibitor and benzoylcholine as a substrate under standard conditions, were able to differentiate between the different genetic variants of this polymorphic enzyme. They found that most of the population (97\%) had the "usual" phenotype and were dibucaine sensitive with around an $80 \%$ inhibition, dubbed the dibucaine number (DN) of 80 . About $3 \%$ of the population had an "intermediate" phenotype with a DN around 62, and an "atypical" phenotype of 1 in 4000 with a DN around 20. The DN appeared to be a constant characteristic for any one individual. Although the dibucaine number has been extensively used by employing different techniques and procedures (Garry and Routh, 1965; Doutin and Brodeur, 1970; Silk et al., 1979; Arnold, 1981; Gottardis et al., 1990; Palleschi et al., 1990; Holownia et al., 1995), still little is known about the mode of inhibition of this enzyme by dibucaine. Cholinesterases have an affinity for cationic substrates and inhibitors, particularly those that have a positively-charged nitrogen atom that is linked to methyl or ethyl groups. They also combine with the enzyme at two different sites as follows; an anionic site that binds the positive charge on the substrate or inhibitor, and an esteratic site that binds the carboxyl group of the ester linkage that is to be broken. SChE has three specific substrates-benzoylcholine, butrylcholine, butrylthiocholine, and many nonspecific substrates including acetylthiocholine (Silk et al., 1979). In the present study, we investigated the kinetics of dibucaine 
inhibition of $\mathrm{SChE}$ in the presence of a specific substrate, BuTch, and a nonspecific substrate, AcTch, so that we could clarify some aspects of the dibucaine inhibition of SChE and gain some insight of the enzyme-binding site.

\section{Materials and Methods}

Butrylthiocholine (BuTch) and benzoylcholine were purchased from Fluka Chemie AG (Buchs, Switzerland). Acetylthiocholine (AcTch) was from BDH Chemicals Ltd. (Poole, England). Quinidine sulfate was from Winlab Limited (Berkshire, United Kingdom). Dibucaine and 5,5'-dithiobis-[2-nitrobenzoic acid] (DTNB) were purchased from the Sigma Chemical Co. (St. Louis, USA). All of the other chemicals were of analytical grade. Plasma from healthy individuals was obtained fresh from donors to the blood bank of the King Khalid University Hospital, Riyadh.

Dibucaine number determinations DN was determined as described by Kalow and Genest (1957). The assay mixture consisted of a $66.7 \mathrm{mM}$ phosphate buffer ( $\mathrm{pH} 7.4$ ), $50 \mu \mathrm{M}$ benzoylcholine, and $20 \mu \mathrm{l}$ plasma. The total volume was $4 \mathrm{ml}$. The reaction was conducted for $3 \mathrm{~min}$ at $37^{\circ} \mathrm{C}$. The reaction was terminated by the addition of quinidine sulfate. The absorbence with or without the addition of $10 \mu \mathrm{M}$ dibucaine, was read at $240 \mathrm{~nm}$. The percent inhibition was then calculated, which was equivalent to the $\mathrm{DN}$.

Enzyme assay SChE activities were measured spectrophotmetrically according to the method of Garry and Routh (1965). Initial velocities were determined at $37^{\circ} \mathrm{C}$ by using $20 \mu \mathrm{l}$ plasma samples as the enzyme source that was added to a $4.5 \mathrm{ml}$ Tris-saline buffer ( $\mathrm{pH}$ 7.4) that contained $50 \mathrm{mM}$ Tris, $11.4 \mathrm{mM}$ $\mathrm{NaCl}$, and $0.252 \mathrm{mM}$ DTNB. Different concentrations of BuTch or AcTch were added to the assay mixture as required. The resulting color read at $412 \mathrm{~nm}$. Determinations were carried out in duplicate, and the activities were measured after $5 \mathrm{~min}$. The reaction was linear during this period. Blanks that contained all of the compounds except the enzyme were used to eliminate any spontaneous hydrolysis of the substrate. A unit of enzyme activity was defined as the amount of enzyme that liberates $1 \mu$ mole-SH groups per minute per ml plasma.

Kinetic studies Enzyme-kinetic measurements were carried out to elucidate the action of dibuciane on the SChE activity and BuTch- or AcTch-SChE interactions. Enzyme assay experiments were performed in the absence or presence of various concentrations of dibucaine $(0-10 \mu \mathrm{M})$ as a function of different substrate (BuTch or AcTch) concentrations (10-200 $\mu \mathrm{M})$. The nature of inhibition that was produced by each set of experiments was determined by analysis of the double-reciprocal plots of the initial velocity versus BuTch or AcTch concentrations. The primary or secondary replots were obtained using the computer program Grafit (Leathebarrow, 1992).

\section{Results}

In the present study the plasma samples that were used had

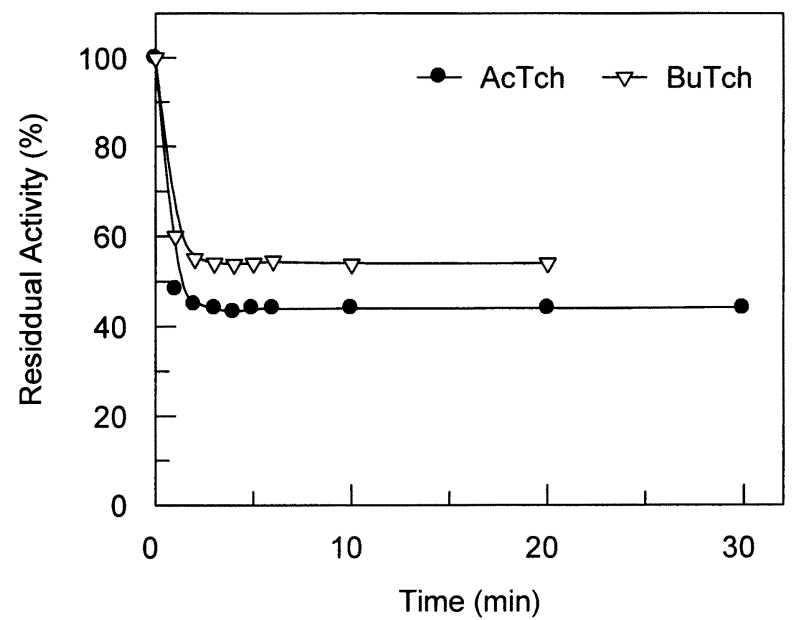

Fig. 1. Time dependence of the inhibition of human SChEcatalyzed hydrolysis of BuTch $(\nabla)$ or AcTch $(\mathbf{O})$ at $200 \mu \mathrm{M}$ each in the presence of $5 \mu \mathrm{M}$ dibucaine. Each point represents the average of 4 determinations with an experimental error of $3 \%$.

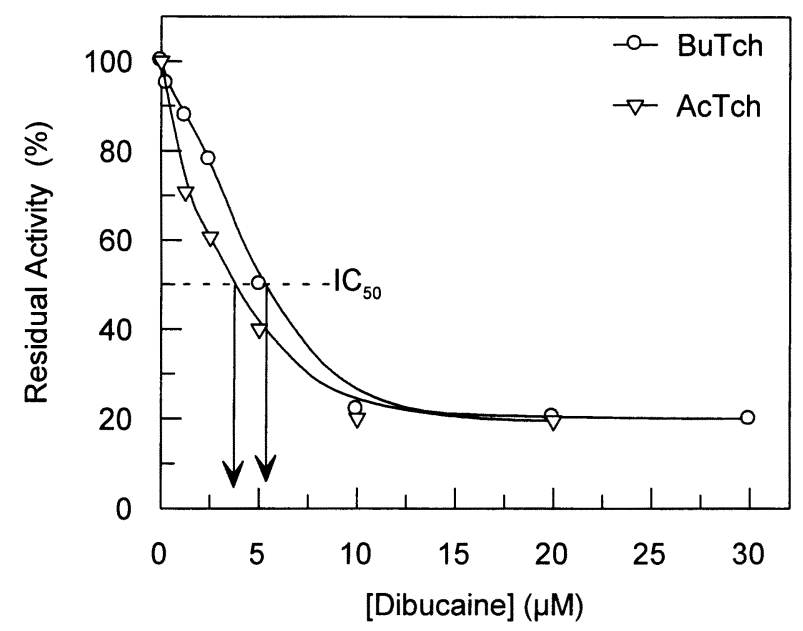

Fig. 2. Inhibition of human SChE activity by varying concentrations of dibucaine $(1.25-30 \mu \mathrm{M})$ in the presence of $200 \mu \mathrm{M}$ of either BuTch $(\bigcirc)$ or AcTch $(\nabla)$. Each point is an average of 3-5 measurements with an experimental error of 3\%.

the "usual" SChE type with a DN between 78-82\%. The inhibitory effects of any one dibucaine concentration on SChE in the presence of either the specific substrate BuTch or the non-specific substrate AcTch, were found to reach maximum within 2 min of incubation and then remain constant (Fig. 1). Dibucaine $(1.25-20 \mu \mathrm{M})$ inhibits SChE activity in the presence of BuTch by $12-78 \%$, or AcTch by $30-80 \%$, in a concentration-dependent manner with an $\mathrm{IC}_{50}$ of $5.3 \mu \mathrm{M}$ and $3.8 \mu \mathrm{M}$, respectively (Fig. 2).

Lineweaver-Burk analysis of dibucaine effects It appears from the Lineweaver-Burk plot of SChE activity in the presence of varying concentrations of dibucaine $(0-10 \mu \mathrm{M})$, 


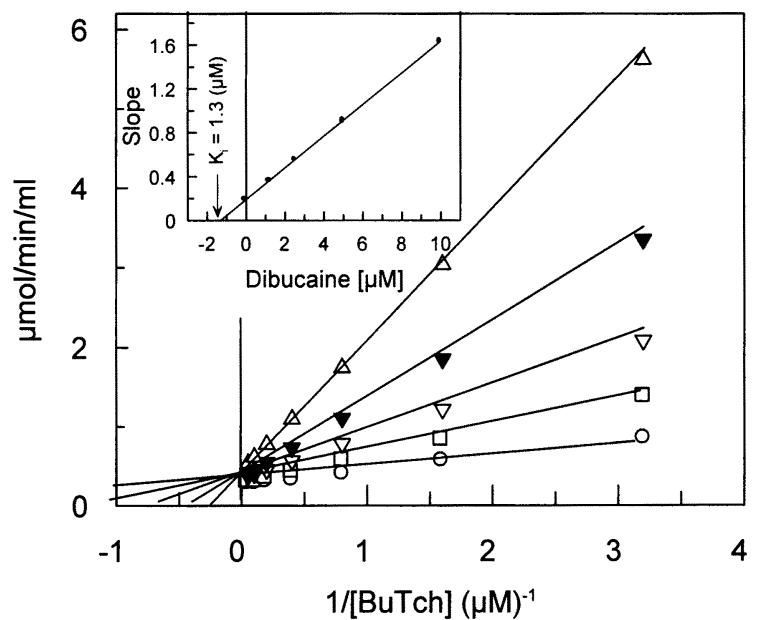

Fig. 3. Kinetic analysis of dibucaine inhibition of the human SChE-catalyzed hydrolysis of BuTch. The data are represented as a double-reciprocal plot of the initial rate of $\mathrm{SH}$ production as a function of the BuTch concentration $(3.13-200 \mu \mathrm{M})$ in the absence $(\bigcirc)$ and presence of $1.25 \mu \mathrm{M}(\square) ; 2.5 \mu \mathrm{M}(\nabla) ; 5 \mu \mathrm{M}$ $(\nabla)$ and $10 \mu \mathrm{M}(\triangle)$ dibucaine. Each point is an average of 3-5 measurements with an experimental error of $3 \%$. Inset shows a secondary replot of the slope values that were derived from the primary double-reciprocal plot vs. dibucaine concentration.

and BuTch as the substrate, that dibucaine increases $K_{m}(220-$ $1000 \%$ ) with no effect on $V_{\max }$ in a competitive type of inhibition (Fig. 3). The inset of the same figure shows a replot of the slopes of the Lineweaver-Burk plots against the dibucaine concentrations that produce an inhibition constant $\left(K_{i}\right)$ of $1.3 \mu \mathrm{M}$. However, these results were obtained with similar concentrations of dibucaine, and AcTch showed an increase in $K_{m}(200-3850 \%)$ and a decrease in $V_{\max }(35-78 \%)$ in a mixed-type inhibition, competitive/noncompetitive (Fig. 4 A). The replots of the slopes and intercepts of the lines against the dibucaine concentrations (Fig. 4 A, inset and Fig. 4 B, respectively) gave straight lines with a $K_{i}$ of $0.66 \mu \mathrm{M}$ for the competitive and a $K_{I}$ of $2.5 \mu \mathrm{M}$ for the noncompetitive inhibitions.

\section{Discussion}

The $\mathrm{K}_{\mathrm{m}}$ value with the specific substrate BuTch was $4.55 \mu \mathrm{M}$, which is comparable to that of the other specific substrate benzoylcholine of $5 \mu \mathrm{M}$ that was obtained by Lockridge and La Du (1978), and less than the $24 \mu \mathrm{M}$ that was reported by Froment et al. (1998). The $K_{m}$ with the non-specific substrate AcTch was about twice that of the specific substrate at $9.1 \mu \mathrm{M}$.

The dibucaine-inhibited SChE enzyme in the presence of either BuTch or AcTch substrates and its inhibitory effects reached a maximum within $2 \mathrm{~min}$. It then remained constant (Fig. 1). This is an indication of its reversible nature (Dawson, 1990). Dibucaine was a less potent inhibitor with the specific
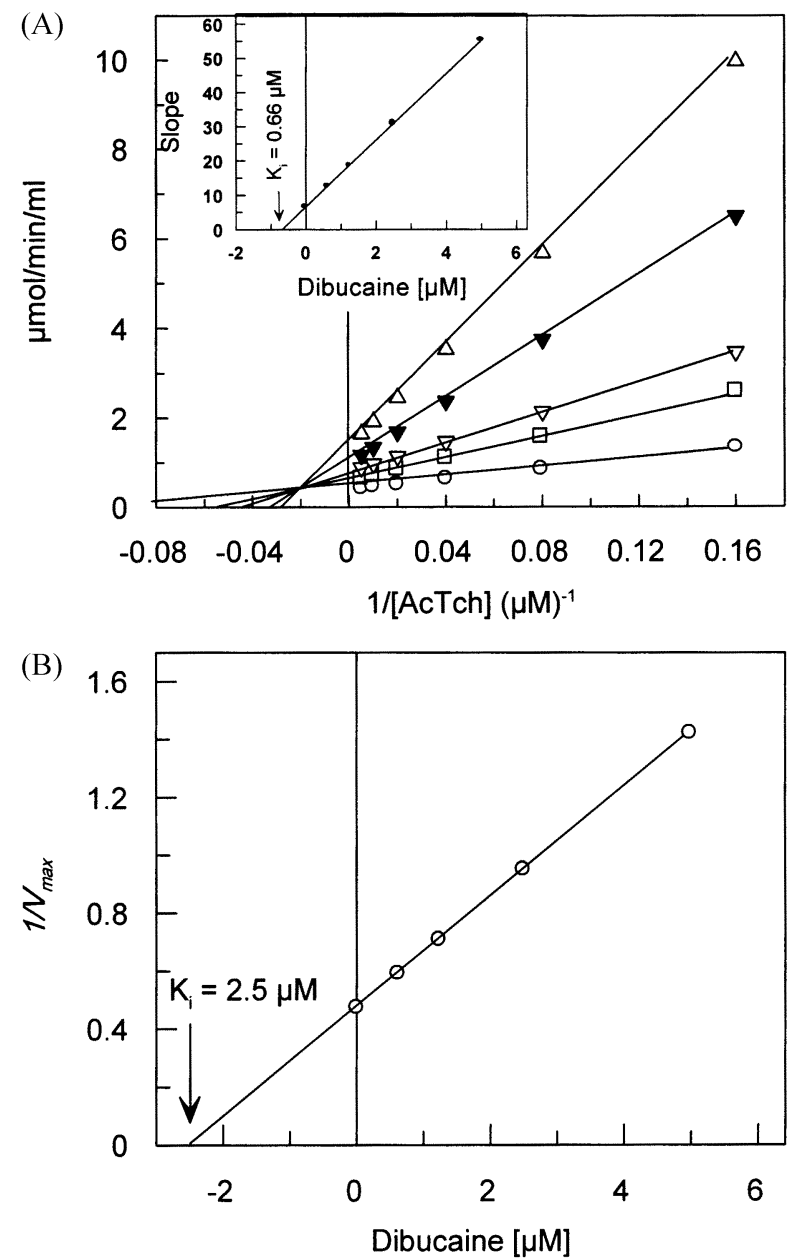

Fig. 4. Kinetic analysis of dibucaine inhibition of the human SChE-catalyzed hydrolysis of AcTch $(6.25-200 \mu \mathrm{M})$. (A) All of the conditions are essentially as described for Figure 3. (B) Secondary replot of $1 / N_{\max }$ values derived from the primary double-reciprocal plot $v s$. dibucaine concentrations for the determination of $K_{I}$.

substrate BuTch than with the nonspecific substrate ATch with $\mathrm{IC}_{50}$ values of $5.3 \mu \mathrm{M}$ and $3.8 \mu \mathrm{M}$, respectively. The inhibitory effect with BuTch had a competitive nature. This is shown by the double-reciprocal plot (Fig. 3). There was no change in $V_{\max }$ and an increase in $K_{m}$, reaching about 10 times with $10 \mu \mathrm{M}$ dibucaine. The replots of the line slopes of the same figure versus the dibucaine concentration gave a straight line that does not pass through the origin. Therefore, the inhibition is partially competitive rather than pure competitive where it should pass through the origin (Segel, 1975). The inhibitory effects of dibucaine in the presence of AcTch were of the linear-mixed type, partially competitive and pure noncompetitive inhibitions with inhibitory constants of $0.66 \mu \mathrm{M}$ and $2.5 \mu \mathrm{M}$, respectively. The replots of the intercepts of the lines in Figure 4A against the dibucaine concentration gave a straight line (Fig. 4B). This indicates a pure noncompetitive inhibition rather than a partial 
noncompetitive inhibition that is indicated by a hyperbolic line (Segel, 1975). Dibucaine may bind at a peripheral-binding site that would prohibit the proper positioning of AcTch at the anionic site and lead to a conformational change that may perturb the esteratic site as well, which is similar to the effect of a related compound, tetrcaine, on acetylcholinesterase activity (Sussman et al., 1991).

Dibucaine, BuTch, and AcTch all have the quaternary ammonium nature; therefore, they would bind to the anionicbinding site of the enzyme (Kalow and Davies, 1958; Rippol et al., 1993). However, dibucaine possesses a butoxy group that is similar to the acyl butryl group of BuTch. It may then compete at another, nearby hydrophobic site. Vellom et al., (1993), using site-specific mutagenesis, was able to substitute $\mathrm{Phe}_{295}$ and $\mathrm{Phe}_{297}$ in the acyl pocket of acetylcholineterase (it shares a 51-54\% sequence homology with SChE) to the corresponding amino acids in SChE, $\mathrm{Leu}_{286}$, and $\mathrm{Ile}_{288}$, respectively. This mutation enhanced the acetycholinesterase activity to BuTch and diminished its activity to AcTch. Therefore, dibucaine may not only compete at the anionicbinding site, but also at this acyl pocket. This may explain the difference in the mode of inhibition with AcTch as the substrate, which is only short by an ethyl group from BuTch. It appears that dibucaine has twice the chance of competing with AcTch than with BuTch, as inferred by the present data of the competitive-inhibition values of $0.66 \mu \mathrm{M}$ and $1.33 \mu \mathrm{M}$, respectively. The presence of an acyl-binding pocket may also explain the enzyme-substrate specificity, where two binding sites are possible for the specific substrate, BuTch $\left(K_{m}=\right.$ $4.55 \mu \mathrm{M})$, and only one for the nonspecific substrate, AcTch $\left(K_{m}=9.1 \mu \mathrm{M}\right)$. The present results agree with the findings of Vellom et al (1993) on the existence of an additional hydrophobic acyl-binding site for SChE.

The original use of dibucaine at the specified concentration of $10^{-5} \mathrm{M}$ (Kalow and Genest, 1957; then also many others) for the determination of DN was apparently a successful choice for routine surveys of enzyme phenotypes. In the present study, $10 \mu \mathrm{M}$ dibucaine was found to be the minimum concentration that gave a maximum inhibition of $80 \%$ with the "usual" SChE isozyme in the presence of either the specific substrate BuTch or the nonspecific substrate AcTch, the same as benzoylcholine. This inhibition remained constant above the specified dibucaine concentration. It was, therefore, unaffected by the small variations in application by the different laboratories and methodologies.

Acknowledgments This work was supported by the Research Center, College of Science, KSU, Grant Bio/1418/ 13. The author would like to thank A. A. Gambo for the technical assistance and M.A. Ismail for assisting in the graphics.

\section{References}

Arnold, W. P. (1981) A rapid, semi-automated method for determining dibucaine numbers. Anesthesiol. 55, 676-679.

Cattozzo G, Franzini C and Rettondini M. (1993) Dibucaine number measured with the Ektachem. Clin. Chem. 39, 15451546.

Cook, D. R., Stiller, R. L. and Weakly, J. N. (1989) In vitro metabolism of mivacurium chloride (BW B1090U) and succinylcholine. Anaesthes. Analges. 68, 452-456.

Dawson, R. M. (1990) Reversibility of the inhibition of acetylcholinesterase by tarcine. Neurosci. Lett. 188, 85-87.

Doutin, D. and Brodeur, J. (1970) An automated method for simultaneous determination of serum pseudocholinesterase activity, dibucaine number and fluoride number. Clin. Biochem. 3, 245-254.

Fleming, N. W., Macres, S., Antognini, J. F. and Vengco, J. (1996) Neuromuscular blocking action of suxamethonium after antagonism of suxamethonium after antagonism of vecuronium by edrophonium, pyridostigmine or neostigmine. $\mathrm{Br} . \mathrm{J}$. Anaesthes. 77, 492-495.

Froment, M.T., Lockridge, O. and Masson, P. (1998) Resistance of butyrylcholinesterase to inactivation by ultrasound: effects of ultrasound on catalytic activity and subunit association. Biochim. Biophys. Acta 1387, 53-64.

Garry, P. J. and Routh, J. I. (1965) A micro method for serum cholinesterase. Clin. Chem. 11, 91-96.

Goedde, H. W., Altland, K. and Schloot, W. (1968) Therapy of prolonged apnea after suxamethonium with purified pseudocholinesterase. New data on kinetics of the hydrolysis of succinyldicholine and succinylomonocholine and further data $\mathrm{N}$-acetyltransferase-polymorphism. Ann. NY Acad. Sci. 151, 742-752.

Gottardis, M., Schmoigl, C., Mitterschiffthaler, G., Mutz, N., Benzer, A., Schobersberger, W., Reitter, B. and Jarosch, E. (1990) The automated determination of the dibucaine number using the Greiner G450 selective analyzer. A routine parameter of significance? Anaesthesist 39, 505-507.

Hersh, L. B., Raj, P. P. and Ohlweiler, D. (1974) Kinetics of succinylcholine hydrolysis by serum cholinesterase: comparison to dibucaine and succinylcholine numbers. J. Pharmocol. Exp. Ther. 189, 544-549.

Hobbiger, F. and Peck, A. W. (1969) Hydrolysis of suxamethonium by different types of plasma. Br. J. Pharmacol. 37, 258-271.

Holownia, P., Newman, D. J., Bruno, C., LaGamba, P., Gerrits, M., Salemink, G., Ossani, M. and Price, C. P. (1995) Automated dibucaine number measurement with DuPont Dimention ES and AR analyzers. Clin. Chem. 41, 664-667.

Kalow, W. and Davies, R. O. (1958) The activity of various esterase inhibitors towards atypical human serum cholinesterase. Biochem. Pharmacol. 1, 183-192.

Kalow, W. and Genest, K. (1957) A method for detection of atypical forms of human serum cholinesterase. Detection of dibucaine numbers. Can. J. Biochem. Physiol. 35, 339-341.

Leathebarrow R. J. (1992) Grafit Version 3.0. Erithacus Software Ltd., Staines, UK.

Lehmann, H. and Silk, E. (1953) Succinylcholine. Br. Med. J. 1, 767-768.

Lockridge, O. and La Du, B. N. (1978) Comparison of atypical and usual human serum cholinesterase. J. Biol. Chem. 253, 361-366.

Masson, P., Froment, M. T., Fortier, P. L., Visicchio, J. E., Bartels, 
C. F. and Lockridge, O. (1998) Butrylcholinesterase-catalyzed hydrolysis of asprin, a negatively-charged ester, and asprinrelated neutral esters. Biochim. Biophys. Acta 1387, 41-52.

Mitchell, J. B. and Harrop-Griffiths, W. (1994) Suxamethonium apnoea and the isolated leg. Anaesthesia 49, 315-316.

Ostergard, D., Jensen, F. S., Jensen, E., Skovgaard, L. T. and Viby-Mogensen, J. (1992) Influence of plasma cholinesterase on recovery from mivacurium-induced neuromuscular blockade in phenotypically normal patients. Acta Anaesthes. Scand. 36, 702-706.

Palleschi, G., Lavagnini, M. G., Moscone, D., Pilloton, R., D'Ottavio, D. and Evangelisti, M. E. (1990) Determination of serum cholinesterase activity and dibucaine numbers by an amperometric choline sensor. Biosens Bioelectron. 5, 27-35.

Ripoll, D. R., Faeman, C. H., Axelsen, P. H., Silman, I. and Sussman, J. L. (1993) An electrostatic mechanism for substrate guidance down the aromatic gorge of acetylcholinesterase. Proc. Natl. Acad. Sci. USA 90, 5128-5132.

Segel, I. H. (1975) Enzyme Kinetics: Behavior and Analysis of Rapid Equilibrium and Steady-state Enzyme Systems. John
Wiley \& Sons, Inc. New York.

Silk, E. King, J. and Whittaker, M. (1979) Assay of cholinesterase in clinical chemistry. Anal. Clin. Biochem. 16, 57-75.

Sockalingam, I. and Green, D. W. (1995) Mivacurium-induced prolonged neuromuscular block. Br. J. Anaesthes. 74, 234-236.

Sussman, J. L., Harel, M., Frolow, F., Oefner, C., Goldman, A., Tokler, L. and Silman, I. (1991) Atomic structure of acetylcholinesterase from Torpedo California: A prototypic acetylcholine-binding protein. Science 253, 872-879.

Thompson, J. C. and Wittaker, M. A. (1966) Study of the pseudocholinesterase in 78 cases of apnoea following suxamethonium. Acta Genet. Stat. Med. 16, 209-222.

Vellom, D. C., Radic, Z., Li, Y., Pickering, N. A., Camp, S. and Taylor, P. (1993) Amino acid residues controlling acetylcholinesterase and butrylcholinesterase specificity. Biochemistry 32, 12-17.

Viby-Mogensen, J. (1981) Succinylcholine neuromuscular blockade of subjects homozygous for atypical plasma cholinesterase. Anesthesiol. 55, 429-34. 